KIAS-P06018

hep-th/0605247

\title{
Comments on Heterotic Flux Compactifications
}

\author{
Tetsuji Kimura* and Piljin $\mathrm{Yi}^{\dagger}$ \\ School of Physics, Korea Institute for Advanced Study, \\ 207-43, Cheongryangri-Dong, Dongdaemun-Gu, Seoul 130-722, Korea
}

\begin{abstract}
In heterotic flux compactification with supersymmetry, three different connections with torsion appear naturally, all in the form $\omega+a H$. Supersymmetry condition carries $a=-1$, the Dirac operator has $a=-1 / 3$, and higher order term in the effective action involves $a=1$. With a view toward the gauge sector, we explore the geometry with such torsions. After reviewing the supersymmetry constraints and finding a relation between the scalar curvature and the flux, we derive the squared form of the zero mode equations for gauge fermions. With $\mathrm{d} H=0$, the operator has a positive potential term, and the mass of the unbroken gauge sector appears formally positive definite. However, this apparent contradiction is avoided by a no-go theorem that the compactification with $H \neq 0$ and $\mathrm{d} H=0$ is necessarily singular, and the formal positivity is invalid. With $\mathrm{d} H \neq 0$, smooth compactification becomes possible. We show that, at least near smooth supersymmetric solution, the size of $H^{2}$ should be comparable to that of $\mathrm{d} H$ and the consistent truncation of action has to keep $\alpha^{\prime} R^{2}$ term. A warp factor equation of motion is rewritten with $\alpha^{\prime} R^{2}$ contribution included precisely, and some limits are considered.
\end{abstract}

\footnotetext{
*tetsuji@kias.re.kr

${ }^{\dagger}$ piljin@kias.re.kr
} 


\section{Introduction}

Flux compactification of superstring theory has emerged as a new and promising framework for bridging the gap between string theory and the real world. A large number of supersymmetric and non-supersymmetric vacua are deemed to exist for generic compactifications to four dimensions [1, 2, 3, 4, and with much reduced number of moduli fields [5, 6]. A prototype of flux compactification was first studied by Becker and Becker [7] in a warped Calabi-Yau compactification scenario in Mtheory. A similarly simple form of flux compactification was found and explored in depth in IIB theory [8, 9, where a large class of solutions were found to be also warped Calabi-Yau compactifications. This model simulates Randall-Sundrum geometry [1] as a bona-fide string theory solution, and has lead to a number of interesting low energy physics [12].

One step away from the warped Calabi-Yau examples, the analysis becomes quite involved. Often the structure of compact manifold is beyond the reach of familiar techniques. Typically the manifold is not even Kähler and the complex structure could be non-integrable [13. Recent progress in understanding of generalized complex geometry and generalized Calabi-Yau geometry [14, 15, 16, 17, 18, 19, 20] will hopefully provide effective tools. However, many properties of the manifold, in particular global properties including topological aspects, remain inaccessible.

Another common difficulty lies in that the compact manifold with flux is singular at leading supergravity level. This follows from an argument of Maldacena and Nuñez 21. This is not necessarily a big disadvantage in type II theories, since the singularity necessary for unbroken supersymmetry would be orientifold planes. We know how to treat these locally in the weak coupling limit. This is in contrast to heterotic theory or M-theory, where we do not have a weak coupling description of such singularity. Fortunately however, the heterotic theory behaves somewhat differently. For one thing, the anomaly condition for $H$ has to involve interaction terms of $\alpha^{\prime} R^{2}$ order for consistency [22], and thus opens up a possibility that we must consider higher derivative interactions at least in some limited sense [23].

Heterotic flux compactification was first studied by Strominger some twenty years ago [24]. While the geometry turns out to be non-Kähler, the relation between $H$ and the complex structure is tightly constrained by the supersymmetry, and one has 
a reasonably concrete geometric characterization. Heterotic examples would be very attractive in part because of its potentially very rich gauge structure. Some limited subfamily may be analyzed via U-duality to F-theory configurations [5, 25, 26], but study of gauge sector in heterotic flux compactification remains largely unaddressed.* From supergravity approach, the conventional tools involve either solving for explicitly spinor zero modes or counting index of some Dirac operator, in order to establish low energy gauge sector. With $\mathcal{N}=1$ supersymmetry in four dimensions, this can address in principle the symmetry breaking pattern and the surviving four-dimensional gauge symmetry, the charge matter field content thereof, and also moduli fields associated with the gauge bundle over the compact directions.

With flux, however, adapting these tools finds difficulties. Even what used to be a trivial task of isolating the zero mode responsible for four-dimensional gaugino, is non-trivial if we take a direct approach by considering the zero mode equation. Our aim here in part is to point out some of such problems, and characterize them. One issue is whether and when the no-go theorem of Maldacena-Nuñez type is effective and when it is not. This issue is more important in heterotic theories because, unlike the case of type IIB, the gauge sector arises from bulk. Reading-off low energy spectrum will depend on details of singularities on the manifold.

Section 2 explains the supersymmetry constraints of Strominger, and the relations among flux, torsion, complex structures and dilaton. From these we find a simple relation between the Ricci scalar curvature and the flux. Section 3 concentrates on Dirac operators whose zero mode solutions generate four-dimensional gaugino and matter fermions. The squared Dirac operators are computed and found to contain spin-dependent potentials in general. In section 4, we employ a simplifying assumption of $\mathrm{d} H=0$, which further reduces the torsion-dependent part of the potential to be spin-independent and positive definite. Naively this would suggest that fourdimensional gauge sector is absent. However, $\mathrm{d} H=0$ and $H \neq 0$ automatically implies a singular internal manifold, so that the gauge sector is closely tied to the singularity of the internal manifold in case of minimal embedding. In section 5, we abandon the $\mathrm{d} H=0$ condition and explore a general equation of motion. In particu-

\footnotetext{
${ }^{*}$ Recently a compactification with $\mathcal{N}=1$ supersymmetry with flux was offered by Yau and collaborators [27, 28], where the internal geometry is a $T^{2}$ fibered over a conformally deformed $K 3$ and completely smooth.
} 
lar, we show that a consistent truncation of the low energy effective action must keep $\alpha^{\prime} R^{2}$ term, which is sometimes ignored on account of $\alpha^{\prime}$ expansion. In the process, we isolate the equation of motion which replaces the one responsible for the no-go theorem of $\mathrm{d} H=0$ case, in a relatively simple form. This naturally leads us to hope for smooth internal manifold for generic supersymmetric flux compactifications, in contrast to the type II counterparts. In the appendices, we list our convention, collect supersymmetry conditions and its known consequence, and also derive a couple identities used in the main part of the paper. In the last appendix, we comment on Atiyah-Singer index densities for smooth manifolds with torsion.

\section{Flux, Torsion, and Curvatures}

Let us first review the supersymmetric flux compactification of heterotic strings on six-manifold $\mathcal{M}_{6}$. Assuming no gaugino condensates [29] $]^{\dagger}$, Strominger obtained a set of supersymmetric conditions on the metric, dilaton, and the Kalb-Ramond field $B$. Here we summarize this set of conditions and obtain further useful identities one can derive from this system.

To set our convention, let us start with the bosonic part of the supergravity/superYang-Mills action in ten dimensions: ${ }^{\ddagger}$

$$
\begin{aligned}
\mathscr{L}=\frac{1}{4} \sqrt{-G} \mathrm{e}^{-2 \Phi}[ & R(\omega)-\frac{1}{3} H_{M N P} H^{M N P}+4\left(\nabla_{M} \Phi\right)^{2} \\
& \left.-\alpha^{\prime}\left\{\operatorname{tr}\left(F_{M N} F^{M N}\right)-\operatorname{tr}\left(R_{M N}\left(\omega_{+}\right) R^{M N}\left(\omega_{+}\right)\right)\right\}\right],
\end{aligned}
$$

This can be obtained from Bergshoeff et.al. 23] via the following map

$$
\begin{aligned}
\left.\phi^{-3}\right|_{\mathrm{BdR}} & =\mathrm{e}^{-2 \Phi},\left.\quad H_{M N P}\right|_{\mathrm{BdR}}=\frac{\sqrt{2}}{3} H_{M N P}, \\
\left.\omega_{M}{ }^{A B}\right|_{\mathrm{BdR}} & =-\omega_{M}^{A B},\left.\quad \lambda\right|_{\mathrm{BdR}}=\sqrt{2} \lambda .
\end{aligned}
$$

Normalization of gravity multiplet is slightly different from the usual one, which can be adjusted by resuscitating ten-dimensional gravitational constant and Yang-Mills coupling constant.

\footnotetext{
${ }^{\dagger}$ See Refs. 30, 31, 32, for compactification with both fermion condensates and flux.

${ }^{\ddagger}$ Our convention is closest to that of Ref. [33. The only difference is in the definition of the dilaton.
} 
Supersymmetry implies existence of a spinor on the six-manifold $\mathcal{M}_{6}$ which solves

$$
\delta \psi_{M}=\nabla_{M}^{(-)} \epsilon=0,
$$

where $\nabla_{M}^{(-)}$is a covariant derivative with a torsionful connection. The metric in string frame has no warp factor,

$$
G_{M N} \mathrm{~d} x^{M} \mathrm{~d} x^{N}=\eta_{\mu \nu} \mathrm{d} x^{\mu} \mathrm{d} x^{\nu}+g_{m n} \mathrm{~d} y^{m} \mathrm{~d} y^{n}
$$

with a metric $g_{m n}$ on the compact manifold $\mathcal{M}_{6}$. We assign $\omega$ to the spin connection of $g_{m n}$. The torsionful connection is defined in terms of $\omega$ shifted by $H$ such as

$$
\omega_{ \pm M}^{A B}=\omega_{M}^{A B} \pm H_{M}^{A B} .
$$

The covariant derivative $\nabla_{M}^{(-)}$is defined with respect to the spin connection $\omega_{-}$. The Einstein frame metric differs from the string frame one by a factor of dilaton,

$$
G_{M N}^{\mathrm{E}} \mathrm{d} x^{M} \mathrm{~d} x^{N}=\mathrm{e}^{-\Phi / 2}\left(\eta_{\mu \nu} \mathrm{d} x^{\mu} \mathrm{d} x^{\nu}+g_{m n} \mathrm{~d} y^{m} \mathrm{~d} y^{n}\right)
$$

so the physically relevant warp factor is $\mathrm{e}^{-\Phi / 2}$.

From a bilinear of $\epsilon$, one constructs an almost complex structure $J_{m n}$, with respect to which the metric $g_{m n}$ is hermitian. Vanishing of dilatino variation, $\delta \lambda=0$, demands that the Nijenhuis tensor vanishes;

$$
0=N_{m n}^{p}=J_{m}{ }^{q} \nabla_{[q} J_{n]}{ }^{p}-J_{n}{ }^{q} \nabla_{[q} J_{m]}{ }^{p},
$$

where we wrote the covariant derivative in place of the ordinary derivatives. Furthermore, the supersymmetry condition (2.3) implies that $J$ is covariantly constant with respect to the torsionful connection

$$
\nabla_{m}^{(-)} J_{n p}=0
$$

This generalizes Kähler conditions. Furthermore, an integrability condition from supersymmetry variations implies a vanishing Ricci two-form

$$
R_{m n}^{a b}\left(\omega_{-}\right) J_{a b}=0
$$

with the curvature associated with $\omega_{-}$. This condition implies an $S U(3)$-structure on the internal manifold $\mathcal{M}_{6}$, and would have implied a Ricci flat condition if there were no torsion. 
These conditions relate the complex structure $J$, the dilaton $\Phi$, and the antisymmetric tensor $H$. First, $H$ can be identified with the so-called Bismut torsion 34]

$$
H_{m n p}=\frac{3}{2} J_{m}^{q} J_{n}{ }^{r} J_{p}^{s} \nabla_{[q} J_{r s]}
$$

and the dilaton is related to $J$ as

$$
\nabla_{m} \Phi=\frac{3}{4} J^{n p} \nabla_{[m} J_{n p]} .
$$

The relation between dilaton and $H$ can be also read off from the above,

$$
\nabla_{m} \Phi=-\frac{1}{2} J_{m n} J_{p q} H^{n p q}
$$

and tells us that the non-primitive part of $H$ is fully encoded in $\mathrm{d} \Phi$.

Recall that a $p$-form on $d$-dimensional background is primitive [35] with respect to an integrable complex structure $J$, if it belongs to a spin $|(d / 2-p) / 2|$ representation under an $S U(2)_{J}$ algebra whose three operators are

$$
\begin{aligned}
& L_{+}=J \wedge \\
& \left.L_{-}=J\right\lrcorner \\
& L_{3}=\frac{(p-d / 2)}{2} .
\end{aligned}
$$

For $p \leq d / 2$, thus, a $p$-form is primitive if and only if it is annihilated by the lowering operator, that is to say, the contraction with $J$ is null. Decomposing $H$ into irreducible representations under $S U(2)_{J}$, we find

$$
H_{m n p}=H_{m n p}^{0}+\frac{3}{2} J_{[m n} J_{p]}^{q} \nabla_{q} \Phi,
$$

where $H^{0}$ has a null contraction with $J$.

The integrability condition (2.9) would have implied Ricci flat condition when $H=0$. With the torsion, it will instead express the Ricci scalar in term of $H$ and $\Phi$. For this, let us contract equation (2.9) with one more $J$,

$$
0=R_{a b m n}\left(\omega_{-}\right) J^{a b} J^{m n} .
$$

We reorganize the right hand side as

$$
3 R_{p[q m n]}\left(\omega_{-}\right) J^{p q} J^{m n}-\left\{R_{p m n q}\left(\omega_{-}\right)+R_{p n q m}\left(\omega_{-}\right)\right\} J^{p q} J^{m n} .
$$


Since the spin connection $\omega_{-}$preserves the complex structure, the latter two pieces both produce a Ricci scalar

$$
-\left\{R_{p m n q}\left(\omega_{-}\right)+R_{p n q m}\left(\omega_{-}\right)\right\} J^{p q} J^{m n}=2 R\left(\omega_{-}\right)=2\left\{R(\omega)-H_{m n p} H^{m n p}\right\},
$$

where in the last step we invoked

$$
R_{m n}^{p q}\left(\omega_{-}\right)=R_{m n}^{p q}(\omega)-2 \nabla_{[m} H_{n]}^{p q}+2 H_{r[m}^{p} H_{n]}^{r q} .
$$

On the other hand, $R_{p[q m n]}\left(\omega_{-}\right)$is entirely made of the torsion part, since $R_{p[q m n]}(\omega)=$ 0 . After some tedious computation, ${ }^{\S}$ we find that (2.15) can be simplified to (up to an overall factor of 2)

$$
0=R(\omega)+\frac{1}{3} H_{m n p} H^{m n p}+6 \nabla_{m} \nabla^{m} \Phi-8\left(\nabla_{m} \Phi \nabla^{m} \Phi\right) .
$$

Later we will use this type of equations to constrain smooth compactifications with flux.

As an easy example, let us note that this last equation alone can be used to show that a simple toroidal compactification is impossible unless $H=0$. For this, note that

$$
0=\mathrm{e}^{-4 \Phi / 3} R(\omega)+\frac{1}{3} \mathrm{e}^{-4 \Phi / 3} H_{m n p} H^{m n p}-\frac{9}{2} \nabla_{m}^{2} \mathrm{e}^{-4 \Phi / 3},
$$

which, with $R(\omega)=0$, implies

$$
\int_{\mathcal{M}_{6}} \mathrm{e}^{-4 \Phi / 3} H_{m n p} H^{m n p}=0
$$

because the last term is a total derivative and integrates to zero. Thus, smooth supersymmetric compactification on a Ricci flat manifold necessarily implies $H=0$. We will come back to this type of vanishing arguments time and again in various contexts.

\section{Zero Mode Equations for 4D Gauge Sector}

Generally speaking, the simplest way of approaching the gauge sector is to look at gaugino. With $\mathcal{N}=1$ supersymmetry unbroken, the low energy spectrum gauge

\footnotetext{
$\S$ See appendix B.3
} 
fermions should encode all information about massless gauge sector, namely unbroken gauge groups, charged matter contents, and the moduli associated with the gauge bundles. In smooth compactifications without flux, and in some orbifold examples, the index theorem for Dirac operators are powerful tools in analyzing fermion sector and, due to the supersymmetry, their bosonic partners [10].

With flux compactification, all kind of new problems show up. In the absence of flux, the zero mode underlying the four-dimensional gaugino field would be identified with the internal part of the supersymmetry parameter $\epsilon$. With flux, however, this simple construction seems no longer possible. $\epsilon$ is a covariantly constant spinor with respect to $\omega_{-}=\omega-H$, yet the connection that appears in the Dirac equation is $\hat{\omega}=\omega-H / 3$, as we will see shortly. While $\omega_{-}$reemerges in the squared form of the Dirac operator, it is still true that $\epsilon$ cannot solve the zero mode equation for the four-dimensional gaugino field unless the torsion vanishes identically. With $\mathcal{N}=1$ supersymmetry unbroken, there should be exactly one zero mode responsible for the four-dimensional gaugino, yet the relevant equation does not tell us this immediately.

In this section we will study the zero mode equation from ten-dimensional gauge sector, in the hope that it will shed some further light on flux compactification in the heterotic theories. The full gaugino equation of motion is quite involved

$$
\begin{aligned}
0= & \not D \chi-\frac{1}{12} H_{M N P} \Gamma^{M N P} \chi \\
& -\nabla_{M} \Phi \Gamma^{M} \chi+3 \Gamma^{M} \Gamma^{N P} F_{N P}\left(\psi_{M}+\frac{2}{3} \Gamma_{M} \lambda\right) .
\end{aligned}
$$

However, rescaling the gaugino field by $\mathrm{e}^{\Phi}$, and then decomposing the gaugino to zero mode $\chi^{0}$ along the compact $\mathcal{M}_{6}$ and four-dimensional gaugino $\Psi$

$$
\chi \sim \mathrm{e}^{\Phi} \chi^{0} \otimes \Psi
$$

we have a simplified zero mode equation along $\mathcal{M}_{6}$

$$
0=\not D(\omega, A) \chi^{0}-\frac{1}{12} H_{m n p} \Gamma^{m n p} \chi^{0}+3 \Gamma^{m} \Gamma^{n p} F_{n p}(\cdots) .
$$

Incorporating the $H$-term into the definition of the covariant derivative, we find that the relevant torsionful connection in this Dirac equation is

$$
\hat{\omega}=\omega-\frac{1}{3} H .
$$


Then, we have

$$
0=\not D(\hat{\omega}, A) \chi^{0}+3 \Gamma^{m} \Gamma^{n p} F_{n p}(\cdots)
$$

The gauge field $A_{m}$ in the Dirac operator acts on $\chi^{0}$ as an adjoint representation of the ten-dimensional gauge group, while its field strength $F_{m n}$ should match its gauge index with that of $\chi^{0}$. Note that the inhomogeneous term in the Dirac equation above involves $F_{n p}$, the background field strength of the gauge bundle on $\mathcal{M}_{6}$. The ten-dimensional gauge group is expected to be broken to satisfy this Bianchi identity for $H$, which forces an non-trivial gauge bundle. We decompose the gauge algebra as

$$
\mathcal{G}=\mathcal{F} \oplus \mathcal{F}_{\perp},
$$

where $F_{n p}$ takes value in the subalgebra $\mathcal{F}$. The unbroken gauge algebra $\mathcal{H}$ is the part of $\mathcal{F}_{\perp}$ that commutes with $\mathcal{F}$,

$$
\mathcal{F}_{\perp}=\mathcal{H} \oplus \mathcal{Q}, \quad[\mathcal{H}, \mathcal{F}]=0
$$

The leftover piece $\mathcal{Q}$ may be expressed as representations under $\mathcal{F} \oplus \mathcal{H}$. Under the above decomposition of the ten-dimensional gauge algebra, the low energy gauge group is generated from $\mathcal{H}$ while the charged matter sector resides in $\mathcal{Q}$.

Since fermions of low energy gauge sectors resides in $\mathcal{F}_{\perp}$ which is orthogonal to $F_{n p}$ in the background, we may drop the inhomogeneous terms linear in gravitino and dilatino fields, ${ }^{\boldsymbol{\top}}$ and we recover a familiar-looking Dirac operators for low energy gauge sector.

$$
0=\not D(\hat{\omega}, A) \chi_{\mathcal{H} \oplus \mathcal{Q}}^{0}
$$

Furthermore, since $\mathcal{H}$ commutes with $\mathcal{F}$, the gaugino zero mode equation does not see the gauge bundle at all, and is the simplest,

$$
0=\not D(\hat{\omega}) \chi_{\mathcal{H}}^{0}
$$

Massless charged matter fermions would be orthogonal to $\mathcal{F}$ but not necessarily commute with it, so we have

$$
0=\not D\left(\hat{\omega}, A_{\mathcal{Q}}\right) \chi_{\mathcal{Q}}^{0}
$$

\footnotetext{
IThey would be important for moduli counting, however.
} 
where we put the subscript $\mathcal{Q}$ on the background gauge field $A_{m}$ to emphasize that it is in the representation $\mathcal{Q}$ under $\mathcal{F}$. Its field strength will be similarly denoted as $F_{m n}^{\mathcal{Q}}$

Note that $\hat{\omega}$ is neither $\omega_{-}$nor $\omega_{+}$. The torsion part $\hat{\omega}$ differs from that of $\omega_{-}$by the factor $1 / 3$, which may look somewhat strange. This factor $1 / 3$ becomes more palatable once we evaluate $\not D^{2}$

$$
\Delta_{\mathcal{H}} \equiv-[\not D(\hat{\omega})]^{2}, \quad \Delta_{\mathcal{Q}} \equiv-\left[\not D\left(\hat{\omega}, A_{\mathcal{Q}}\right)\right]^{2}
$$

We have

$$
\Delta_{\mathcal{H}}=-\Gamma^{m} D_{m}(\hat{\omega}) \Gamma^{n} D_{n}(\hat{\omega})=-\frac{1}{\sqrt{g}} D_{m}\left(\omega_{-}\right) g^{m n} \sqrt{g} D_{n}\left(\omega_{-}\right)+V,
$$

with the potential $V$

$$
V=\frac{1}{4}\left\{R(\omega)-\frac{1}{3} H_{m n p} H^{m n p}+\frac{1}{12}(\mathrm{~d} H)_{m n p q} \Gamma^{m n p q}\right\} .
$$

When commuting $\Gamma^{n}$ through $D_{m}(\hat{\omega})$, one obtains terms linear in $D_{m}(\hat{\omega})$. Of these, the piece with Christoffel connection conspires to generate the explicit metric factors in the $D(\hat{\omega})^{2}$ piece, while the torsion-piece is absorbed into $D^{2}$ piece by shifting $D_{m}(\hat{\omega})$ into $D_{m}\left(\omega_{-}\right)$and completing a square.

The first term, $D^{2}$ type, is a Laplace operator on the (Lie-algebra-valued) spinor bundle, so we may as well write

$$
\Delta_{\mathcal{H}}=-[\not D(\hat{\omega})]^{2}=\nabla_{m}\left(\omega_{-}\right)^{\dagger} \nabla^{m}\left(\omega_{-}\right)+V
$$

provided that the manifold is smooth and compact. Similarly, we have

$$
\Delta_{\mathcal{Q}}=-\left[\not D\left(\hat{\omega}, A_{\mathcal{Q}}\right)\right]^{2}=\nabla_{m}\left(\omega_{-}, A_{\mathcal{Q}}\right)^{\dagger} \nabla^{m}\left(\omega_{-}, A_{\mathcal{Q}}\right)+V+\frac{i}{2} F_{m n}^{\mathcal{Q}} \Gamma^{m n}
$$

While we started with the torsion of the amount $-H / 3$, the zero mode equation solves a Laplace-type equation (with a potential) with torsion $-H$ instead [36]. Interestingly, the covariant derivative with torsion $-H$ is precisely the one that appears in supersymmetry condition. 


\section{$4 \mathrm{~d} H=0$ or Minimal Embedding}

Gauge sector of heterotic flux compactification remains relatively obscure. In the usual compactification with $H=0$, the Bianchi identity

$$
\mathrm{d} H=\alpha^{\prime}[\operatorname{tr}\{R(\omega) \wedge R(\omega)\}-\operatorname{tr}(F \wedge F)],
$$

is solved by embedding the $S U(3)$ spin connection to the gauge sector, thereby breaking gauge group down to $E_{6} \times E_{8}$. With flux, the Bianchi identity is replaced by

$$
\mathrm{d} H=\alpha^{\prime}\left[\operatorname{tr}\left\{R\left(\omega_{+}\right) \wedge R\left(\omega_{+}\right)\right\}-\operatorname{tr}(F \wedge F)\right],
$$

with respect to the $S O(6)$-valued curvature two-form $R\left(\omega_{+}\right)$[37. Note that this curvature two-form is made from the connection $\omega+H$, whereas the $S U(3)$-structure of the manifold is associated with $\omega-H$.

A major hurdle in understanding flux compactification of the heterotic string theory is to classify solutions to this twisted Bianchi identity. With $H$-flux, the nearest analog of such a minimal embedding is to set $\mathrm{d} H=0$, by choosing the gauge bundle to have the property

$$
\operatorname{tr}(F \wedge F)=\operatorname{tr}\left\{R\left(\omega_{+}\right) \wedge R\left(\omega_{+}\right)\right\}
$$

Further, the simplest way to do this is to use $\omega_{+}$as the gauge connection again, which makes $\mathrm{d} H=0$ even when $H \neq 0$ carries a topological flux. If one chooses to embed $\omega_{+}$into the gauge bundle, the gauge bundle will be of $S O(6)$-structure group. This would break the gauge group to $S O(10) \times E_{8}$ for $E_{8} \times E_{8}$ heterotic theory, for instance. Concentrating on the broken $E_{8}$ part, the holonomy group and the unbroken gauge group are, respectively,

$$
\mathcal{F}=S O(6), \quad \mathcal{H}=S O(10)
$$

The matter fermions reside in $\mathcal{Q}$ consisting of representations,

$$
(6,10) \oplus(4,16) \oplus(\overline{4}, \overline{16})
$$

under $S O(6) \times S O(10)$. It is unclear to us if there is a solution with $\mathrm{d} H=0$ with $\mathcal{F}$ smaller than $S O(6)$. 
In a background with $\mathrm{d} H=0$, the squared Dirac operator $\not D^{2}$ is simplified further as the potential become spin-independent,

$$
V=\frac{1}{4}\left(R(\omega)-\frac{1}{3} H_{m n p} H^{m n p}\right) .
$$

For further reduction, recall that the analog of the vanishing Ricci two-form condition relates $R(\omega)$ to $H$ and derivatives of $\Phi$ as

$$
R(\omega)=-\frac{1}{3} H_{m n p} H^{m n p}-6 \nabla_{m}^{2} \Phi+8\left(\nabla_{m} \Phi\right)^{2} .
$$

This is still cumbersome because of the second derivative of dilaton. Now, consider the quantity $J \wedge \mathrm{d} H$, and rewrite it as

$$
J \wedge \mathrm{d} H=\mathrm{e}^{2 \Phi} \mathrm{d}\left(\mathrm{e}^{-2 \Phi} J \wedge H\right)-\mathrm{e}^{2 \Phi} \mathrm{d}\left(\mathrm{e}^{-2 \Phi} J\right) \wedge H .
$$

From the form of $H$ and $\Phi$, we have

$$
\mathrm{e}^{2 \Phi} \mathrm{d}\left(\mathrm{e}^{-2 \Phi} J\right)=2 * H
$$

while

$$
\mathrm{e}^{2 \Phi} \mathrm{d}\left(\mathrm{e}^{-2 \Phi} J \wedge H\right)=-*\left(\nabla_{m}^{2} \Phi-2\left(\nabla_{m} \Phi\right)^{2}\right) .
$$

Combining these results we finally have

$$
*(J \wedge \mathrm{d} H)=-\nabla_{m}^{2} \Phi+2\left(\nabla_{m} \Phi\right)^{2}-\frac{1}{3} H_{m n p} H^{m n p}
$$

so that $\mathrm{d} H=0$ then implies that the right hand side vanishes. The two equations, $R_{a b m n}\left(\omega_{-}\right) J^{a b} J^{m n}=0$ and $\mathrm{d} H=0$, together produce a simpler formula for the Ricci scalar,

$$
R(\omega)=\frac{5}{3} H_{m n p} H^{m n p}-4\left(\nabla_{m} \Phi\right)^{2} .
$$

Then $F$-independent "potential term" in the squared Dirac operator becomes

$$
\frac{1}{4}\left(R(\omega)-\frac{1}{3} H_{m n p} H^{m n p}\right)=\frac{1}{3} H_{m n p}^{0} H^{0 m n p}
$$

with the help of the decomposition of $H$ into the primitive part $H^{0}$ and the rest;

$$
H_{m n p} H^{m n p}=\left(H_{m n p}^{0}\right)^{2}+3\left(\nabla_{m} \Phi\right)^{2} .
$$


Thus, the potential term of the operator $-\not D^{2}$ becomes

$$
V=\frac{1}{3} H_{m n p}^{0} H^{0 m n p}
$$

which is positive definite whenever $H^{0}$, the primitive part of the torsion, is non-zero. Zero mode equations are then,

$$
\left[\nabla_{m}\left(\omega_{-}, A_{\mathcal{Q}}\right)^{\dagger} \nabla^{m}\left(\omega_{-}, A_{\mathcal{Q}}\right)+\frac{1}{3} H_{m n p}^{0} H^{0 m n p}+\frac{i}{2} F_{m n} \Gamma^{m n}\right] \chi_{\mathcal{Q}}^{0}=0
$$

and

$$
\left[\nabla_{m}\left(\omega_{-}\right)^{\dagger} \nabla^{m}\left(\omega_{-}\right)+\frac{1}{3} H_{m n p}^{0} H^{0 m n p}\right] \chi_{\mathcal{H}}^{0}=0 .
$$

Note that the latter operator is formally positive definite, as long as $H^{0} \neq 0$. Ordinarily, the last form of zero mode equation would show absence of massless gaugino in four dimensions, and thus by supersymmetry no unbroken gauge group. Recall that a usual vanishing theorem would have followed from

$$
\begin{aligned}
0 & =\int_{\mathcal{M}_{6}} \bar{\chi}_{\mathcal{H}}^{0}\left[\nabla_{m}\left(\omega_{-}\right)^{\dagger} \nabla^{m}\left(\omega_{-}\right)+\frac{1}{3} H_{m n p}^{0} H^{0 m n p}\right] \chi_{\mathcal{H}}^{0} \\
& =\int_{\mathcal{M}_{6}}\left[\left|\nabla_{m}\left(\omega_{-}\right) \chi_{\mathcal{H}}^{0}\right|^{2}+\frac{1}{3}\left|H_{m n p}^{0}\right|^{2}\left|\chi_{\mathcal{H}}^{0}\right|^{2}\right]
\end{aligned}
$$

forcing $\chi_{\mathcal{H}}^{0}=0$. Owing to the supersymmetry, this would also imply that no unbroken gauge sector exists.

However, this assertion must be false; in the constraints coming from the compactification nothing forces the bundle over $\mathcal{M}_{6}$ to be of maximal rank, and a priori, there is no reason why $\mathcal{H}$ should be null. In fact, we expect exactly one zero mode solution to the above zero mode equation of $\Delta_{\mathcal{H}}$. To see the way out of this quandary, note that the above argument is correct only if there is no obstruction to the integration by part into the second line. It is well-known that in type II flux compactification the compact manifold has to be singular, which can be attributed orientifold planes that carries a negative RR charge and a negative tension [8]. In the heterotic case, it must be that, when $\mathrm{d} H=0$, something similar happens and the internal manifold becomes singular.

In fact, using the identity (4.11), one may argue for a no-go theorem. Recall that $*(J \wedge \mathrm{d} H)=0$ implies

$$
0=\nabla_{m}^{2} \Phi-2\left(\nabla_{m} \Phi\right)^{2}+\frac{1}{3} H_{m n p} H^{m n p},
$$


which can be rewritten as

$$
\frac{1}{2} \nabla_{m}^{2} \mathrm{e}^{-2 \Phi}=\frac{1}{3} \mathrm{e}^{-2 \Phi} H_{m n p} H^{m n p}
$$

If there are no boundaries and no singularities in $\mathcal{M}_{6}$, we find that

$$
\frac{1}{3} \int_{\mathcal{M}_{6}} \mathrm{e}^{-2 \Phi} H_{m n p} H^{m n p}=\frac{1}{2} \int_{\mathcal{M}_{6}} \nabla_{m}^{2} \mathrm{e}^{-2 \Phi}=0
$$

forcing $H=0$ and bringing us back to compactification without flux if the internal manifold is assumed to be compact and smooth.

Therefore, there cannot be any regular compactification of heterotic string theory with $H \neq 0$ and $\mathrm{d} H=0$ [38]. This is also related to the no-go theorem of MaldacenaNuñez type [21, 8]. In the latter, the existence of singularity can be seen from the equation of motion for the warp factor; here, it so happens that the warp factor in Einstein frame is precisely $\mathrm{e}^{-\Phi / 2}$. In what would have been the simplest scenario for heterotic theories, things are more complicated. $\mathrm{d} H=0$ forces the gauge sector crucially to depend on understanding of singularities in $\mathcal{M}_{6}$.

On the other hand, the detailed form of (4.17) is suggestive with its positive potential term. For a large internal manifold, the zero mode is no longer uniform and localized away from the region of large $\left|H^{0}\right|^{2}$. Also its behavior must be rather singular near the singularity of the internal manifold. In view of interesting local physics found in type IIB theories [8, 41], the precise form of this zero mode, including its behavior near singularities, deserves further attention. We hope to come back to this problem later.

\section{$5 \mathrm{~d} H \neq 0$ and Smooth Compactifications}

As we saw above, what would have been the simplifying assumption of $\mathrm{d} H=0$, seems to cause more trouble than otherwise. For the supergravity approach, one is thus lead to more generic configurations with non-minimal background with $\mathrm{d} H \neq 0$. Recently an example of smooth compactification was proposed by the authors of Ref. [27, 28], where indeed all examples were non-minimal. In this section, we will consider precisely what equation replaces (4.20) and how the usual no-go theorem is avoided in heterotic theories. Recall that Maldacena-Nuñez type argument would 
be difficult to evade if we stick to an Einstein gravity coupled to a quadratic action of tensor fields. Thus, it has something to do with what truncation of the effective action we are allowed to use in the presence of such a flux that size of $\mathrm{d} H$ is not ignorable compared to that of $H^{2}$.॥

In order to justify the low energy description, the size of compact manifold, must be substantially larger than $\sqrt{\alpha^{\prime}}$, so that expansion in $\alpha^{\prime}$ is justifiable. Let $L$ be the linear size of the internal manifold $\mathcal{M}_{6}$, such that

$$
\frac{\alpha^{\prime}}{L^{2}} \ll 1
$$

In the conventional supergravity approach, one takes the Lagrangian (2.1) but keeps only up to $F^{2}$ term, and argue $R^{2}$ terms is of higher order. For actual supersymmetric solutions, however, this is somewhat misleading, since on-shell values of Ricci scalar and $H^{2}$ are no larger than the higher order term, $\alpha^{\prime} \operatorname{tr}\left(R^{2}\right)$. In fact, we know from general form of supersymmetric solutions above that

$$
H \wedge * H \sim J \wedge \mathrm{d} H \sim \alpha^{\prime} J \wedge[\operatorname{tr}(F \wedge F)-\operatorname{tr}\{R(\omega) \wedge R(\omega)\}]
$$

and also that

$$
J \wedge\{\operatorname{tr}(F \wedge F)-\operatorname{tr}\{R(\omega) \wedge R(\omega)\}\} \sim *\left[\operatorname{tr}\left(F_{m n} F^{m n}\right)-\operatorname{tr}\left\{R_{m n}(\omega) R^{m n}(\omega)\right\}\right] .
$$

From these, one should expect that generally**

$$
F_{m n} \sim \frac{1}{L^{2}} \sim R_{b m n}^{a}(\omega), \quad H_{m n p} \sim \frac{\sqrt{\alpha^{\prime}}}{L^{2}} \sim \nabla_{m} \Phi,
$$

while the Ricci-tensor is of order $\nabla H$ and $H^{2}$ and thus of order $\alpha^{\prime} / L^{4}$

$$
R_{p m}(\omega)=R_{b m n}^{a}(\omega) E_{a}^{m} e_{p}^{b} \sim \frac{\alpha^{\prime}}{L^{4}}
$$

At least for supersymmetric configurations and also nearby non-supersymmetric ones, one must keep $R^{2}$ term in the Lagrangian for consistency. However, the difference between $R_{b m n}^{a}(\omega)$ and $R_{b m n}^{a}\left(\omega_{+}\right)$is roughly of the order

$$
\nabla H \sim H^{2} \sim \frac{\alpha^{\prime}}{L^{4}}
$$

\footnotetext{
"For recent discussions of higher order $\alpha^{\prime}$ correction, see Ref. 39, 40] also.

${ }^{* *}$ Note that, upon restoring the overall gravitational constant $1 / \kappa_{10}^{2}$ in front of the Lagrangian, $H$ has the dimension of mass, while $F$ has dimension of mass squared.
} 
and we may as well use the curvature without torsion $R_{b m n}^{a}(\omega)$ in the last term of (2.1), instead of $R_{b m n}^{a}\left(\omega_{+}\right)$, simplifying computations greatly.

The main message here is that for generic compactification with flux, one cannot drop $\alpha^{\prime} R^{2}$ piece from the action on account of $\alpha^{\prime}$ expansion. The only exception to this is the case of $\mathrm{d} H=0$, and even in that case, the effect of $R^{2}$ terms is cancelled by effect of $F^{2}$ term, rather than being subleading to the rest of terms.

With these in mind, let us consider how the equation (4.20) is related with equations of motion. From the Lagrangian (2.1), one obtains a linear combination of the field equations of the form

$$
0=\left[\frac{\delta}{\delta \Phi}-\frac{1}{2} G^{M N} \frac{\delta}{\delta G^{M N}}\right] \int \mathrm{d}^{10} x \mathscr{L}
$$

which gives

$$
\begin{aligned}
0= & \nabla_{M}^{2} \mathrm{e}^{-2 \Phi}-\frac{2}{3} \mathrm{e}^{-2 \Phi} H_{M N P} H^{M N P} \\
& -\alpha^{\prime} \mathrm{e}^{-2 \Phi}\left(\operatorname{tr}\left(F_{M N} F^{M N}\right)-\operatorname{tr}\left(R_{M N} R^{M N}\right)\right)+\cdots
\end{aligned}
$$

where the ellipsis denotes terms that came from variation of the Riemann tensor with respect to the metric in the quartic term, $R_{P}{ }^{Q M N} \delta R^{P}{ }_{Q M N}$, and is proportional to

$$
\alpha^{\prime} \nabla^{M} \nabla^{N}\left(\mathrm{e}^{-2 \Phi} R_{M P N}^{P}\right)
$$

with an order 1 coefficient.

Using the supersymmetry condition on $F$ and $R$, we have

$$
\operatorname{tr}\left(F_{m n} F^{m n}\right)=-2 *(J \wedge \operatorname{tr}(F \wedge F))
$$

and also up to leading non-vanishing order in $\alpha^{\prime} / L^{2}$ that

$$
\operatorname{tr}\left(R_{m n} R^{m n}\right)=-2 *(J \wedge \operatorname{tr}(R \wedge R)) .
$$

Therefore the above equation may be reorganized for supersymmetric background as

$$
0=\nabla_{m}^{2} \mathrm{e}^{-2 \Phi}-\frac{2}{3} \mathrm{e}^{-2 \Phi} H_{m n p} H^{m n p}-2 \mathrm{e}^{-2 \Phi} *(J \wedge \mathrm{d} H)+\cdots
$$

up to the leading order in $\alpha^{\prime}$, with help of the Bianchi identity for $H$. The total derivative term of the ellipsis drops out since the ordinary Ricci tensor $R_{m n}=R_{m p n}^{p}$ 
is of order $\alpha^{\prime} / L^{4}$, so that

$$
\alpha^{\prime} \nabla^{m} \nabla^{n}\left(\mathrm{e}^{-2 \Phi} R_{m p n}^{p}\right) \sim \frac{\left(\alpha^{\prime}\right)^{2}}{L^{6}} \ll \frac{\alpha^{\prime}}{L^{4}} \sim H^{2} .
$$

The ellipsis in (5.12) may be ignored as far as supersymmetric compactifications (and nearby configurations) are concerned.

Then, $\mathrm{d} H=0$ again implies (4.20)

$$
0=\frac{1}{2} \nabla_{m}^{2} \mathrm{e}^{-2 \Phi}-\frac{1}{3} \mathrm{e}^{-2 \Phi} H_{m n p} H^{m n p} .
$$

Note that this same equation was obtained in two ways; first, by rewriting $J \wedge \mathrm{d} H=0$ with help of supersymmetry conditions, and second, from equation of motion after imposing $(J \wedge \mathrm{d} H)=0$. In the latter, the supersymmetry comes in when we exchanged $\operatorname{tr}|R|^{2}-\operatorname{tr}|F|^{2}$ in favor of $*(J \wedge \mathrm{d} H)$. This is exactly as it should, since supersymmetry implies the equation of motion. Therefore, what replaces (4.20) in more general supersymmetric background is the above combination of the field equation, which we may write more compactly as

$$
\nabla_{m}^{2} \mathrm{e}^{-2 \Phi}=\mathrm{e}^{-2 \Phi}\left[4|H|^{2}+2 \alpha^{\prime}\left(\operatorname{tr}|F|^{2}-\operatorname{tr}|R|^{2}\right)\right]
$$

which is self-consistent and correct up to order $\alpha^{\prime} / L^{4}$.

This clearly shows how the usual no-go theorem against smooth flux compactification is evaded in heterotic theories via the higher curvature term. Also this reiterates the fact that, in order to have a smooth flux compactification, it is necessary to have

$$
\operatorname{tr}|F|^{2} \neq \operatorname{tr}|R|^{2}
$$

and

$$
\int_{\mathcal{M}_{6}} \mathrm{e}^{-2 \Phi}\left[2|H|^{2}+\alpha^{\prime} \operatorname{tr}|F|^{2}\right]=\int_{\mathcal{M}_{6}} \mathrm{e}^{-2 \Phi}\left[\alpha^{\prime} \operatorname{tr}|R|^{2}\right] .
$$

Generically, both sides are of order $\alpha^{\prime} L^{2}$.

The equation (5.15) without $R^{2}$ term has been used to argue that constant dilaton background is necessarily torsion-free. With $R^{2}$ absent, both $H^{2}$ and $F^{2}$ are nonnegative so $\nabla \Phi=0$ will force both $H=0$ and $F=0$. Once we have a non-trivial gauge bundle and a non-trivial compact geometry, though, we cannot drop $R^{2}$ term. Instead, $\nabla \Phi=0$ would enforce a local relation

$$
2|H|^{2}+\alpha^{\prime} \operatorname{tr}|F|^{2}=\alpha^{\prime} \operatorname{tr}|R|^{2}
$$


everywhere on $\mathcal{M}_{6}$ with the primitivity condition $J \wedge H(\sim * \mathrm{~d} \Phi)=0$ also satisfied. A priori, a torsionful compactification with constant $\Phi$ remains an interesting possibility to pursue, although the local condition (5.18) may prove to be difficult to implement. In any case, here, the potential $V$ in the squared Dirac operator degenerates to another simple form

$$
V=-\frac{1}{6} H_{m n p}^{0} H^{0 m n p}+\frac{1}{48}\left(\mathrm{~d} H^{0}\right)_{m n p q} \Gamma^{m n p q},
$$

and we can see that the spin-independent part is now negative definite, in contrast to the $\mathrm{d} H=0$ case.

Another interesting limit is when the primitive part of $H$ vanishes, $H_{m n p}^{0}=0$, upon which we have

$$
|H|^{2}=\frac{1}{3 !} H_{m n p} H^{m n p}=\frac{1}{2}\left(\nabla_{m} \Phi\right)^{2} .
$$

With this the above equation is simplified to

$$
\nabla^{2} \mathrm{e}^{-\Phi}=\alpha^{\prime} \mathrm{e}^{-\Phi}\left[\operatorname{tr}|F|^{2}-\operatorname{tr}|R|^{2}\right]
$$

This case imposes only a global constraint

$$
\alpha^{\prime} \int_{\mathcal{M}_{6}} \mathrm{e}^{-\Phi}\left[\operatorname{tr}|F|^{2}\right]=\alpha^{\prime} \int_{\mathcal{M}_{6}} \mathrm{e}^{-\Phi}\left[\operatorname{tr}|R|^{2}\right] .
$$

It is known [42] that when this happens the geometry becomes conformally Kähler, so that the metric and the two-form

$$
\widetilde{g}=\mathrm{e}^{-\Phi} g, \quad \widetilde{J}=\mathrm{e}^{-\Phi} J
$$

together define a Kähler manifold. In particular, the Einstein metric in such special cases can be written as

$$
G_{M N}^{\mathrm{E}} \mathrm{d} x^{M} \mathrm{~d} x^{N}=\mathrm{e}^{-\Phi / 2} \eta_{\mu \nu} \mathrm{d} x^{\mu} \mathrm{d} x^{\nu}+\mathrm{e}^{\Phi / 2} \widetilde{g}_{m n} \mathrm{~d} y^{m} \mathrm{~d} y^{n}
$$

so the dilaton plays the role of warp factor in a familiar form as in type IIB story with 3-fluxes. For the sake of completeness, we also write the potential $V$ for the squared Dirac operator in this case,

$$
V=\frac{3}{2} \mathrm{e}^{\Phi} \nabla_{m}^{2} \mathrm{e}^{-\Phi}+\frac{1}{48}(\mathrm{~d} H)_{m n p q} \Gamma^{m n p q} .
$$

with $H$ carrying no primitive piece. 


\section{Summary}

We have explored the torsionful geometry of the supersymmetric flux compactification of heterotic string theory. With the aim at understanding how the low energy gauge sector arises, and also trying to understand the flux compactification better, we isolated the zero mode equation of ten-dimensional gaugino field, and showed that zero modes responsible for gaugino and charged matter fermions obey relatively simple elliptic equations. Along the way, we found that $\mathrm{d} H=0$ limit always implies a singular internal manifold, and thus allows the four-dimensional gaugino to exist despite the formally positive internal operator. With $\mathrm{d} H \neq 0$ comparable to $H^{2}$, smooth compactification becomes possible and we argued why this is a generic behavior by showing that the higher order term $\alpha^{\prime} R^{2}$ is comparable to $H^{2}$ and cannot be neglected at least for configurations near supersymmetric compactifications.

As far as counting the matter content of charged fermions is concerned, a lesson we learned is that the old counting of "generations," that is, the number of chiral charged matter fields, cannot be imported to the compactification with flux. Recall that the renowned formula where the generation is given by the Euler number divided by 2 [10], replies on the "minimal embedding" and $\mathrm{d} H=0$. With $H$ flux, singularities compromise naive index formula, at least until we know how to classify and handle the singularities.

Despite the singularity of the manifold, the shape of the zero mode equations when $\mathrm{d} H=0$ is itself suggestive. The primitive part of the torsion supplies a spinindependent non-negative potential to the squared zero mode equation, and its consequence to the local form of the gauge zero modes might be worth pursuing, in view of how local physics with a hierarchical warp factor was important in type IIB compactification.

For more general and non-singular backgrounds $\mathrm{d} H \neq 0$, it remains to understand how to solve the Bianchi identity and what this, together with torsion, implies for index densities in general. Here we took the first step by constructing the "Hamiltonian" $\Delta_{\mathcal{Q}}$, relevant for the counting of chiral fermions. We hope to come back to study of the index densities, in relation with the anomaly condition, in near future. ${ }^{\dagger \dagger}$

\footnotetext{
${ }^{\dagger \dagger}$ See appendix D for comments on existing computations for index densities with torsion.
} 


\section{Acknowledgement}

We are indebted to Seok Kim for many useful discussions. PY was supported in part by the Science Research Center Program of the Korea Science and Engineering Foundation through the Center for Quantum Spacetime(CQUeST) of Sogang University with grant number R11-2005-021.

\section{Appendix}

\section{A Conventions}

Conventions for indices are as follows:

$$
\begin{aligned}
M, N, \ldots & \text { real ten-dimensional coordinate indices, } \\
A, B, \ldots & \text { real ten-dimensional } S O(9,1) \text { indices } \\
\mu, \nu, \ldots & \text { real four-dimensional coordinate indices, } \\
m, n, \ldots & \text { real six-dimensional coordinate indices, } \\
a, b, \ldots & \text { real six-dimensional } S O(6) \text { indices }
\end{aligned}
$$

Antisymmetrization of the indices is defined as

$$
T_{\left[M_{1} M_{2} \cdots M_{p}\right]}=\frac{1}{p !}\left(T_{M_{1} M_{2} \cdots M_{p}}-T_{M_{2} M_{1} \cdots M_{p}} \pm \text { permutations }\right) .
$$

We adopt the following rule about the contraction of tensors:

$$
\left|F_{p}\right|^{2}=\frac{1}{p !} g^{M_{1} N_{1}} g^{M_{2} N_{2}} \cdots g^{M_{p} N_{p}} F_{M_{1} \cdots M_{p}} F_{N_{1} \cdots N_{p}} .
$$

The $p$ ! cancels the sum over permutations of the indices, so that each independent component appears with coefficient 1.

Vielbeins $e_{M}{ }^{A}$ and their inverses $E_{A}{ }^{M}$ from the curved spacetime metric $g_{M N}$ and the tangent space metric $\eta_{A B}$ are such that

$$
\begin{aligned}
g_{M N} & =\eta_{A B} e_{M}{ }^{A} e_{N}{ }^{B}, & \eta_{A B} & =g_{M N} E_{A}{ }^{M} E_{B}{ }^{N}, \\
\delta_{M}^{N} & =e_{M}{ }^{A} E_{A}{ }^{N}, & \delta_{A}^{B} & =E_{A}{ }^{M} e_{M}{ }^{B} .
\end{aligned}
$$


Here are more conventions and identities related to differential forms on a $D$-dimensional Riemannian manifold $\mathcal{M}_{D}$ [43]:

$$
\begin{gathered}
\omega_{p}=\frac{1}{p !} \omega_{M_{1} \cdots M_{p}} \mathrm{~d} x^{M_{1}} \wedge \cdots \wedge \mathrm{d} x^{M_{p}} \\
* \omega_{p}=\frac{\sqrt{\left|g_{D}\right|}}{p !(D-p) !} \varepsilon_{N_{p+1} \cdots N_{D}}{ }^{M_{1} \cdots M_{p}} \omega_{M_{1} \cdots M_{p}} \mathrm{~d} x^{N_{p+1}} \wedge \cdots \wedge \mathrm{d} x^{N_{D}} \\
* 1=\sqrt{\left|g_{D}\right|} \mathrm{d} x^{1} \wedge \cdots \wedge \mathrm{d} x^{D} \\
* * \omega_{p}=(-1)^{p(D-p)} \omega_{p}, \\
g_{D} \varepsilon^{M_{1} \cdots M_{p}}{ }_{N_{p+1} \cdots N_{D}} \cdot \varepsilon_{M_{1} \cdots M_{p}}{ }^{L_{p+1} \cdots L_{D}}=p !(D-p) ! \cdot \delta_{\left[N_{p+1}\right.}^{L_{p+1}} \cdots \delta_{\left.N_{D}\right]}^{L_{D}}
\end{gathered}
$$

where $\varepsilon_{M_{1} \cdots M_{D}}$ and $\varepsilon^{M_{1} \cdots M_{D}}$ are tensor densities.

Finally we close with a useful identity among the Dirac matrices, which is needed for computation of $-\not D^{2}$.

$$
\begin{aligned}
\Gamma^{A_{1} A_{2} \cdots A_{p}} \Gamma_{B_{1} B_{2} \cdots B_{q}} & \\
& =\sum_{k=0}^{\min (p, q)}(-1)^{\frac{1}{2} k(2 p-k-1)} \frac{p ! q !}{(p-k) !(q-k) ! k !} \delta_{\left[B_{1}\right.}^{\left[A_{1}\right.} \cdots \delta_{B_{k}}^{A_{k}} \Gamma^{\left.A_{k+1} \cdots A_{p}\right]}{ }_{\left.B_{k+1} \cdots B_{q}\right]} .
\end{aligned}
$$

where

$$
\Gamma^{A_{1} \cdots A_{p}}=\frac{1}{p !}\left(\Gamma^{A_{1}} \Gamma^{A_{2}} \cdots \Gamma^{A_{p}}-\Gamma^{A_{2}} \Gamma^{A_{1}} \cdots \Gamma^{A_{p}} \pm \text { permutations }\right) .
$$

\section{B Supersymmetry and an $S U(3)$-structure}

Here we summarize the supersymmetry variations of fermions with zero-th order in $\alpha^{\prime}$ (the higher order corrections are shown in [23]):

$$
\begin{aligned}
\delta \psi_{M} & =\left\{\partial_{M}+\frac{1}{4} \omega_{-M}{ }^{A B} \Gamma_{A B}\right\} \epsilon \\
\delta \lambda & =-\frac{1}{4}\left\{\Gamma^{M} \nabla_{M} \Phi-\frac{1}{6} H_{M N P} \Gamma^{M N P}\right\} \epsilon, \\
\delta \chi & =-\frac{1}{4} F_{M N} \Gamma^{M N} \epsilon .
\end{aligned}
$$

\section{B.1 Invariant Forms}

In the heterotic supergravity, we assign the chiralities of fermions with the followings:

$$
\Gamma_{(10)} \psi_{M}=+\psi_{M}, \quad \Gamma_{(10)} \chi=+\chi, \quad \Gamma_{(10)} \lambda=-\lambda, \quad \Gamma_{(10)} \epsilon=+\epsilon .
$$


The ten-dimensional supersymmetry parameter $\epsilon$, which is a Majorana-Weyl spinor, decomposes into two kinds of Weyl spinors under $\operatorname{Spin}(9,1) \rightarrow \operatorname{Spin}(3,1) \times \operatorname{SU}(4)$

$$
\epsilon=f \cdot \xi_{+} \otimes \eta_{+}+f^{*} \cdot \xi_{-} \otimes \eta_{-}
$$

where the complex conjugates of these two Weyl spinors are assigned such as $\left(\xi_{+}\right)^{*}=$ $\xi_{-}$and $\left(\eta_{+}\right)^{*}=\eta_{-}$, respectively; $f$ and $f^{*}$ are complex scale factors depending on coordinates. In this paper we fix these coefficients to 1 . The Weyl spinors on the six-manifold $\mathcal{M}_{6}$ define an invariant two-form $J$ and an invariant three-form $\Omega$ :

$$
\begin{gathered}
\nabla_{m}^{(-)} \eta_{ \pm}=0, \quad \nabla_{m}^{(-)} J_{a b}=\nabla_{m}^{(-)} \Omega_{a b c}=0 \\
\eta_{ \pm}^{\dagger} \eta_{ \pm}=1, \quad J_{a b}=-i \eta_{+}^{\dagger} \Gamma_{a b} \eta_{+}, \quad \Omega_{a b c}=\eta_{+}^{\mathrm{T}} \Gamma_{a b c} \eta_{+} .
\end{gathered}
$$

Via the Fierz identity on the Weyl spinors in six-dimensional space, one can identify $J_{m}{ }^{n}$ with the almost complex structure and finds that the metric on the sixdimensional space becomes hermitian with respect to this almost complex structure:

$$
J_{m}^{p} J_{p}^{n}=-\delta_{m}^{n}, \quad J_{m}^{p} J_{n}^{q} g_{p q}=g_{m n} .
$$

Since there are no invariant five-forms and there should be one volume form on the $S U(3)$-structure manifold, these invariant forms satisfy the following equations

$$
J \wedge \Omega=0, \quad J \wedge J \wedge J=\frac{3 i}{4} \Omega \wedge \bar{\Omega}=3 ! \sqrt{|g|} \mathrm{d} y^{1} \wedge \cdots \wedge \mathrm{d} y^{6} .
$$

This $\Omega$ is not a holomorphic three-form, however. See next subsection.

\section{B.2 Geometry of Supersymmetric Compactifications}

Supersymmetry variations on the six-manifold $\mathcal{M}_{6}$ restrict the geometrical conditions via relations among the fields $\{\Phi, H, F\}$ and the geometrical quantities $\{J, \Omega\}$. The most typical conditions are given by

$$
\begin{aligned}
0 & =R^{a b}{ }_{m n}\left(\omega_{-}\right) J_{a b} & & \left(\text { from } \delta \psi_{m}=0\right), \\
J_{[m}{ }^{q} \nabla_{|q|} J_{n p]} & =-2 J_{\left[m^{q}{ }^{q}{ }_{n}{ }^{r} H_{p] q r}\right.} & & \left(\text { from } \delta \psi_{m}=0\right), \\
N_{m n p} & =H_{m n p}-3 J_{[m}{ }^{q} J_{n}{ }^{r} H_{p] q r} & & \left(\text { from } \delta \psi_{m}=0\right), \\
N_{m n p} & =0 & & (\text { from } \delta \lambda=0),
\end{aligned}
$$




$$
\begin{aligned}
H_{m n p} J^{n p} & =2 J_{m}{ }^{q} \nabla_{q} \Phi & & (\text { from } \delta \lambda=0) \\
0 & =F_{m n} J^{m n} & & (\text { from } \delta \chi=0) .
\end{aligned}
$$

By using these we further obtain various simple conditions among the fields and geometrical quantities in terms of the differential forms [33]:

$$
\begin{aligned}
0 & =-2 \mathrm{~d} \Phi+\theta, \\
H & =T^{(\mathrm{B})}=-\frac{1}{2} * \mathrm{e}^{+2 \Phi} \mathrm{d}\left(\mathrm{e}^{-2 \Phi} J\right), \\
0 & =\mathrm{d}\left(\mathrm{e}^{-2 \Phi} * J\right)=\frac{1}{2} \mathrm{~d}\left(\mathrm{e}^{-2 \Phi} J \wedge J\right), \\
0 & =\mathrm{d}\left(\mathrm{e}^{-2 \Phi} \Omega\right),
\end{aligned}
$$

which implies that there is a holomorphic three-form

$$
\bar{\partial}\left(\mathrm{e}^{-2 \Phi} \Omega\right)=0 .
$$

Some of quantities above are well-known mathematical objects for complex geometry. In addition to the familiar Nijenhuis tensor $N_{m n}{ }^{p}$, the Lee-form $\theta$ and the Bismut torsion $T_{m n p}^{(\mathrm{B})} 34$ are defined as

$$
\begin{aligned}
\theta & \equiv J\lrcorner \mathrm{d} J=\frac{3}{2} J^{m n} \nabla_{[m} J_{n p]} \mathrm{d} y^{p}, \\
N_{m n}{ }^{p} & \equiv J_{m}^{q} \nabla_{[q} J_{n]}{ }^{p}-J_{n}{ }^{q} \nabla_{[q} J_{m]}{ }^{p}, \\
T_{m n p}^{(\mathrm{B})} & \equiv \frac{3}{2} J_{m}{ }^{q} J_{n}{ }^{r} J_{p}{ }^{s} \nabla_{[s} J_{q r]}=-\frac{3}{2} J_{[m}{ }^{q} \nabla_{|q|} J_{n p]} .
\end{aligned}
$$

A useful identity for $\mathrm{d} H$ can be found as follows. Let us decompose $H$-flux on the internal space into the primitive part $H^{0}$ and the non-primitive part like

$$
\left.H=H^{0}+\frac{1}{4} J \wedge K, \quad J\right\lrcorner H^{0}=J \wedge H^{0}=0,
$$

where $K_{m} \equiv H_{m n p} J^{n p}=2 J_{m}{ }^{n} \nabla_{n} \Phi$ given by the supersymmetry variation (B.6e). By using the equations (B.7b), (B.7c) and $J \wedge H^{0}=0$, we evaluate the followings:

$$
\begin{aligned}
J \wedge \mathrm{d} H & =\mathrm{e}^{2 \Phi} \mathrm{d}\left(\mathrm{e}^{-2 \Phi} J \wedge H\right)-\mathrm{e}^{2 \Phi} \mathrm{d}\left(\mathrm{e}^{-2 \Phi} J\right) \wedge H \\
\mathrm{e}^{2 \Phi}\left(\mathrm{de}^{-2 \Phi} J\right) \wedge H & =-2 H \wedge * H=(* 1) \frac{1}{3} H_{m n p} H^{m n p} \\
\mathrm{e}^{2 \Phi} \mathrm{d}\left(\mathrm{e}^{-2 \Phi} J \wedge H\right) & =\frac{1}{4} \mathrm{e}^{2 \Phi} \mathrm{d}\left(\mathrm{e}^{-2 \Phi} J \wedge J \wedge K\right) \\
& =\frac{1}{2} \mathrm{~d} K \wedge * J=-*\left(\nabla_{m}^{2} \Phi-2\left(\nabla_{m} \Phi\right)^{2}\right) .
\end{aligned}
$$


Thus we obtain an equation among the invariant two-form $J$, the $H$-flux and the dilaton $\Phi$ such as

$$
*(J \wedge \mathrm{d} H)=-\nabla_{m}^{2} \Phi+2\left(\nabla_{m} \Phi\right)^{2}-\frac{1}{3} H_{m n p} H^{m n p} .
$$

\section{B.3 Ricci Scalar Curvature}

Here we summarize the computation that gives (2.19). Starting with

$$
0=R_{m n}^{a b}\left(\omega_{-}\right) J^{m n}
$$

where $\omega_{-}=\omega-H$, so that

$$
R_{p m q n}\left(\omega_{-}\right)=R_{p m q n}(\omega)-\nabla_{q} H_{p m n}+\nabla_{n} H_{p m q}+H_{p r q} H_{m n}^{r}-H_{p r n} H_{m q}^{r} .
$$

Contracting with the complex structure one more time,

$$
0=R_{p q m n}\left(\omega_{-}\right) J^{p q} J^{m n}=3 R_{p[q m n]}\left(\omega_{-}\right) J^{p q} J^{m n}+2 R_{p m q n} J^{p q} J^{m n},
$$

The first piece is purely a torsion

$$
\begin{aligned}
3 R_{p[q m n]}\left(\omega_{-}\right) J^{p q} J^{m n}= & 6\left(-\nabla_{[m} H_{|p| q n]}+H_{p r[m} H_{q n]}^{r}\right) J^{p q} J^{m n} \\
= & -6 J^{p q} J^{m n} \nabla_{m} H_{p q n}+2 J^{p q} J^{m n} H_{p q r} H_{m n}{ }^{r} \\
& +4 J^{p q} J^{m n} H_{p r m} H_{q n}^{r},
\end{aligned}
$$

while the second is the Ricci scalar with torsion

$$
\begin{aligned}
2 R_{\text {pmqn }}\left(\omega_{-}\right) J^{p q} J^{m n} & =2 R_{p m q n}\left(\omega_{-}\right) g^{p q} g^{m n} \\
& =2\left(R(\omega)-H_{m n p} H^{m n p}\right) .
\end{aligned}
$$

Relations between $J, H$ and the dilaton can be used to show

$$
\begin{aligned}
J^{p q} J^{m n} H_{p r m} H_{q n}^{r} & =-\frac{1}{3} H_{m n p} H^{m n p}, \\
J^{p q} J^{m n} H_{p q r} H_{m n}{ }^{r} & =4\left(\nabla_{m} \Phi\right)^{2}, \\
J^{p q} J^{m n} \nabla_{m} H_{p q n} & =-2 \nabla_{m}^{2} \Phi-\frac{2}{3} H_{m n p} H^{m n p}+4\left(\nabla_{m} \Phi\right)^{2} .
\end{aligned}
$$

Combining these, we find

$$
\begin{aligned}
\frac{1}{2} R_{p q m n}\left(\omega_{-}\right) J^{p q} J^{m n}= & -3\left(-2 \nabla_{m}^{2} \Phi-\frac{2}{3} H_{m n p} H^{m n p}+4\left(\nabla_{m} \Phi\right)^{2}\right) \\
& +4\left(\nabla_{m} \Phi\right)^{2}-\frac{2}{3} H_{m n p} H^{m n p}+\left(R(\omega)-H_{m n p} H^{m n p}\right) .
\end{aligned}
$$


Thus, the supersymmetry demands the scalar curvature of the internal manifold to satisfy

$$
0=R(\omega)+\frac{1}{3} H_{m n p} H^{m n p}+6 \nabla_{m}^{2} \Phi-8\left(\nabla_{m} \Phi\right)^{2} .
$$

\section{Equations of Motion}

Equations of motion for $\Phi, G_{M N}, B_{M N}$ and $\chi$ in string frame are given as follows:

$$
\begin{aligned}
0= & -R(\omega)+\frac{1}{3} H_{M N P} H^{M N P}+4\left(\nabla_{M} \Phi\right)^{2}-4 \nabla_{M}^{2} \Phi-\mathcal{Z}, \\
0= & R_{M N}(\omega)-H_{M P Q} H_{N}{ }^{P Q}+2 \nabla_{M} \nabla_{N} \Phi \\
- & \frac{1}{2} G_{M N}\left[R(\omega)-\frac{1}{3} H_{P Q R} H^{P Q R}-4\left(\nabla_{P} \Phi\right)^{2}+4 \nabla_{P}^{2} \Phi+\mathcal{Z}\right] \\
- & 2 \alpha^{\prime}\left[\operatorname{tr}\left(F_{M P} F_{N}{ }^{P}\right)-\operatorname{tr}\left\{R_{M P}\left(\omega_{+}\right) R_{N}{ }^{P}\left(\omega_{+}\right)\right\}\right] \\
- & 2 \alpha^{\prime} \mathrm{e}^{2 \Phi}\left[2 \nabla^{P} \nabla_{(+)}^{Q}\left\{\mathrm{e}^{-2 \Phi} R_{M P N Q}\left(\omega_{+}\right)\right\}-\nabla_{(+)}^{Q}\left\{\mathrm{e}^{-2 \Phi} R_{M P Q R}\left(\omega_{+}\right)\right\} H_{N}{ }^{P R}\right. \\
& -2 \nabla^{P}\left\{\mathrm{e}^{-2 \Phi} R_{M P Q R}\left(\omega_{+}\right) H_{N} Q R\right\}-2 \mathrm{e}^{-2 \Phi} R_{M P Q R}\left(\omega_{+}\right) H_{N}{ }^{P S} H_{S} Q R \\
& \left.-\nabla^{P} \nabla_{(+)}^{Q}\left\{\mathrm{e}^{-2 \Phi} R_{M N P Q}\left(\omega_{+}\right)\right\}+\nabla^{P}\left\{\mathrm{e}^{-2 \Phi} R_{M N Q R}\left(\omega_{+}\right) H_{P}{ }^{Q R}\right\}\right],
\end{aligned}
$$

$$
\begin{aligned}
0= & \nabla^{M}\left(\mathrm{e}^{-2 \Phi} H_{M N P}\right), \\
0= & \not D(\omega, A) \chi-\frac{1}{12} \Gamma^{M N P} \chi H_{M N P} \\
& -\Gamma^{M} \chi \nabla_{M} \Phi+\frac{3}{2} \Gamma^{M} \Gamma^{N P}\left(F_{N P}+\hat{F}_{N P}\right)\left(\psi_{M}+\frac{2}{3} \Gamma_{M} \lambda\right),
\end{aligned}
$$

where $\mathcal{Z} \equiv-\alpha^{\prime}\left[\operatorname{tr}\left(F_{M N} F^{M N}\right)-\operatorname{tr}\left\{R_{M N}\left(\omega_{+}\right) R^{M N}\left(\omega_{+}\right)\right\}\right]$.

Notice that we defined the trace with respect to the former two indices of the curvature tensors such as $\operatorname{tr}\left\{R_{M N}\left(\omega_{+}\right) R^{M N}\left(\omega_{+}\right)\right\}=-R_{P Q M N}\left(\omega_{+}\right) R^{P Q M N}\left(\omega_{+}\right)$. Via the anomaly cancellation in ten dimensions, the Bianchi identity of $H$-flux is given by (see [37, 23])

$$
\mathrm{d} H=\alpha^{\prime}\left[\operatorname{tr}\left\{R\left(\omega_{+}\right) \wedge R\left(\omega_{+}\right)\right\}-\operatorname{tr}(F \wedge F)\right] .
$$




\section{Index Densities with Torsion}

It is often stated that introduction of torsion does not affect index. This is natural since the torsion piece, as far as the classical geometry goes, can be thought of a continuous deformation on the Dirac operator, under which an index of Fredholm operator should be invariant.

We should not be mislead to expect from this mathematical statement that flux has no effect in the fermion counting in string compactification. Fluxes in string compactification can affect the fermion counting in two qualitative ways. One is to modify the Dirac equation so that fermions of different kind (or chirality) get mixed up and usual chirality operator cannot be used to define an index. Another, which is relevant for the gauge sector fermions in our heterotic theory, is the fact that the geometry can backreact to the flux in some essential way. This was the case for flux compactification with $\mathrm{d} H=0$, as we saw above.

With these said, it is still curious that index density formula for an arbitrary smooth manifold with torsion seem not available. The closest work to this can be found in [36], which computes the Atiyah-Singer index densities when the manifold has a completely anti-symmetric torsion which is closed. According to this work, the Atiyah-Singer index density for $\not D(\hat{\omega}, A)$ with $\mathrm{d} H=0$ would be given by the usual characteristic polynomial [36, 44]

$$
\mathcal{A}\left(R_{+}\right) \wedge \operatorname{ch}(F)
$$

with A-genus $\mathcal{A}$ and the Chern character $c h$ of the gauge bundle. Note that in place of the curvature two-form, we have the curvature two-form $R_{+}$of the connection $\omega_{+}=\omega+H$, rather than $R_{-}$.

Assuming that the path integral approach taken there is accurate, one may understand switching as follows. Recall that the index density formula is obtained by using the identity

$$
\operatorname{index} \not D \equiv \lim _{\beta \rightarrow \infty}\left(\mathrm{e}^{-\beta \Delta} \prod_{a=1}^{6} \Gamma_{a}\right)=\lim _{\beta \rightarrow 0}\left(\mathrm{e}^{-\beta \Delta} \prod_{a=1}^{6} \Gamma_{a}\right),
$$

which holds provided that the spectrum of $\not D$ is discrete. Realizing $\Delta$ as a Hamiltonian of a supersymmetric quantum mechanics with supercharge $\not D$, one obtains the metricdependent part of the index density from one-loop determinant of bosonic oscillation. 
The curvature part of the index densities are built with monomials like

$$
\operatorname{tr}\left\{R_{a b m}^{n}\left(\omega_{-}\right) e^{a} \wedge e^{b}\right\}^{k}
$$

with vielbein one-forms $e^{a}$. Here the trace is taken over $m$ and $n$ indices, the coordinate indices. Note that this is opposite of usual invariant density where the Lie algebra indices $a$ and $b$ are traced over. Without torsion, this flip does not matter since the two sets of indices are interchangeable. With torsion, instead, we have

$$
R_{m n p q}\left(\omega_{+}\right)=R_{p q m n}\left(\omega_{-}\right)+(\mathrm{d} H)_{p q m n}=R_{p q m n}\left(\omega_{-}\right)
$$

Thus, the formal computation yields an invariant polynomial of type

$$
\operatorname{tr}\left\{R\left(\omega_{+}=\omega+H\right)\right\}^{2 k}
$$

provided that $\mathrm{d} H=0$.

If the manifold were smooth and compact, this would have demonstrated that the index is independent of $H$. To see this, let us define an $S O$-valued torsion one-form as

$$
T_{b}^{a}=H_{m b}^{a} \mathrm{~d} y^{m}
$$

It can be seen easily that

$$
\operatorname{tr}\left\{R\left(\omega_{+}\right)\right\}^{2 k}=\operatorname{tr}\{R(\omega)\}^{2 k}+\mathrm{d}\left(2 k \int_{0}^{1} \mathrm{~d} x \operatorname{tr}\left\{T \wedge R(\omega+x T)^{2 k-1}\right\}\right),
$$

which shows that the torsion contribution will integrate to zero on a compact and smooth manifold.

With $\mathrm{d} H \neq 0$, the quantum mechanics is somewhat modified because of the quartic terms that survives in $\Delta$, in the form $\sim(\mathrm{d} H)_{a b c d} \Gamma^{a b c d}$ [45, 46]. Naive extension of Mavromatos' computation is not difficult to carry out, but it is not clear whether the final formula makes sense. $\mathrm{d} H$ can enter in two distinct ways: First is a further shift of the curvature tensor $R\left(\omega_{+}\right)$to $R\left(\omega_{+}\right)-\mathrm{d} H$. Note that this is because that curvature is actually $R\left(\omega_{-}\right)$with the coordinate indices and the Lie-algebra indices flipped. Also $\mathrm{d} H$ makes appearance as a factor of $\mathrm{d} H / \beta$ outside the trace, where all 4 indices should be regarded as coordinate indices. Contribution like the latter must disappear upon integration on the six-manifold, yet explicit check of this has 
not been performed. In fact, it is not clear if the resulting formula following this line of derivation makes any sense as a topological density.

One reason for such difficulties must be due to the subtlety in the regularization of the path integral approach. Somewhat formal manipulation, originally due to [47, 48, seems to fail for manifold with torsion. In literature, rigorous computations of this kind exists only in the context of four-dimensional spacetime, largely in connection with axial anomaly in quantum field theory. See [49] for detailed and rigorous computation that demonstrates that torsion contributes a total derivative term only in four-dimensional case. For rigorous computation of index densities, a heat-kernel approach [50] would be more desirable which is not yet available for six and higher dimensions.

\section{References}

[1] L. Susskind, "The Anthropic Landscape of String Theory," hep-th/0302219.

[2] M. R. Douglas, "The Statistics of String/M-theory Vacua," JHEP 0305 (2003) 046, hep-th/0303194.

[3] S. Ashok and M. R. Douglas, "Counting Flux Vacua," JHEP 0401 (2004) 060, hep-th/0307049.

[4] F. Denef and M. R. Douglas, "Distributions of Flux Vacua," JHEP 0405 (2004) 072, hep-th/0404116.

[5] K. Dasgupta, G. Rajesh and S. Sethi, "M-theory, Orientifolds and G-flux," JHEP 9908 (1999) 023, hep-th/9908088

[6] S. Kachru, M. B. Schulz and S. Trivedi, "Moduli Stabilization from Fluxes in a Simple IIB Orientifold," JHEP 0310 (2003) 007, hep-th/0201028

[7] K. Becker and M. Becker, "M-theory on Eight-manifolds," Nucl. Phys. B 477 (1996) 155, hep-th/9605053.

[8] S. B. Giddings, S. Kachru and J. Polchinski, "Hierarchies from Fluxes in String Compactifications," Phys. Rev. D 66 (2002) 106006, hep-th/0105097 
[9] I. R. Klebanov and M. J. Strassler, "Supergravity and a Confining Gauge Theory: Duality Cascades and $\chi S B$-resolution of Naked Singularities," JHEP 0008 (2000) 052, hep-th/0007191.

[10] P. Candelas, G. T. Horowitz, A. Strominger and E. Witten, "Vacuum Configurations for Superstrings," Nucl. Phys. B 258 (1985) 46.

[11] L. Randall and R. Sundrum, "A Large Mass Hierarchy from a Small Extra Dimension," Phys. Rev. Lett. 83 (1999) 3370, hep-ph/9905221.

[12] S. Kachru, R. Kallosh, A. Linde and S. P. Trivedi, "De Sitter Vacua in String Theory," Phys. Rev. D 68(2003) 046005, hep-th/0301240.

[13] G. L. Cardoso, G. Curio, G. Dall'Agata, D. Lüst, P. Manousselis and G. Zoupanos, "Non-Kähler String Backgrounds and Their Five Torsion Classes," Nucl. Phys. B 652 (2003) 5, hep-th/0211118.

[14] N. J. Hitchin, "Generalized Calabi-Yau Manifolds," math.DG/0209099.

[15] J. P. Gauntlett, D. Martelli and D. Waldram, "Superstrings with Intrinsic Torsion," Phys. Rev. D 69 (2004) 086002, hep-th/0302158.

[16] S. Fidanza, R. Minasian and A. Tomasiello, "Mirror Symmetric SU(3)structure Manifolds with NS Fluxes," Commun. Math. Phys. 254 (2005) 401, hep-th/0311122.

[17] M. Gualtieri, "Generalized Complex Geometry," math.DG/0401221.

[18] M. Graña, R. Minasian, M. Petrini and A. Tomasiello, "Supersymmetric Backgrounds from Generalized Calabi-Yau Manifolds," JHEP 0408 (2004) 046, hep-th/0406137.

[19] M. Graña, R. Minasian, M. Petrini and A. Tomasiello, "Generalized Structures of $\mathcal{N}=1$ vacua," JHEP 0511 (2005) 020, hep-th/0505212.

[20] M. Graña, "Flux Compactifications in String Theory: A Comprehensive Review," Phys. Rept. 423 (2006) 91, hep-th/0509003. 
[21] J. M. Maldacena and C. Nuñez, "Supergravity Description of Field Theories on Curved Manifolds and a No Go Theorem," Int. J. Mod. Phys. A 16 (2001) 822, hep-th/0007018.

[22] M. B. Green and J. H. Schwarz, "Anomaly Cancellation in Supersymmetric $D=10$ Gauge Theory and Superstring Theory," Phys. Lett. B 149 (1984) 117.

[23] E. A. Bergshoeff and M. de Roo, "The Quartic Effective Action of the Heterotic String and Supersymmetry," Nucl. Phys. B 328 (1989) 439.

[24] A. Strominger, "Superstrings with Torsion," Nucl. Phys. B 274 (1986) 253.

[25] K. Becker, M. Becker, P. S. Green, K. Dasgupta and E. Sharpe, "Compactifications of Heterotic Strings on Non-Kähler Complex Manifolds. II," Nucl. Phys. B 678 (2004) 19, hep-th/0310058.

[26] K. Becker, M. Becker, K. Dasgupta and P. S. Green, "Compactifications of Heterotic Theory on non-Kähler Complex Manifolds. I," JHEP 0304 (2003) 007, hep-th/0301161.

[27] J. X. Fu and S. T. Yau, "The Theory of Superstring with Flux on Non-Kähler Manifolds and the Complex Monge-Ampère Equation," hep-th/0604063.

[28] K. Becker, M. Becker, J. X. Fu, L. S. Tseng and S. T. Yau, "Anomaly Cancellation and Smooth Non-Kähler Solutions in Heterotic String Theory," hep-th/0604137.

[29] M. Dine, R. Rohm, N. Seiberg and E. Witten, "Gluino Condensation in Superstring Models," Phys. Lett. B 156 (1985) 55.

[30] G. Lopes Cardoso, G. Curio, G. Dall'Agata and D. Lüst, "Heterotic String Theory on Non-Kähler Manifolds with H-flux and Gaugino Condensate," Fortsch. Phys. 52 (2004) 483, hep-th/0310021.

[31] A. R. Frey and M. Lippert, "AdS Strings with Torsion: Non-complex Heterotic Compactifications," Phys. Rev. D 72 (2005) 126001, hep-th/0507202. 
[32] P. Manousselis, N. Prezas and G. Zoupanos, "Supersymmetric Compactifications of Heterotic Strings with Fluxes and Condensates," Nucl. Phys. B 739 (2006) 85, hep-th/0511122.

[33] G. L. Cardoso, G. Curio, G. Dall'Agata and D. Lüst, "BPS Action and Superpotential for Heterotic String Compactifications with Fluxes," JHEP 0310 (2003) 004, hep-th/0306088.

[34] J. M. Bismut, "A Local Index Theorem for Non-Kähler Manifolds," Math. Ann. 284 (1989) 681.

[35] S. Gukov, C. Vafa and E. Witten, "CFT's from Calabi-Yau Four-folds," Nucl. Phys. B 584 (2000) 69 [Erratum-ibid. B 608 (2001) 477], hep-th/9906070.

[36] N. E. Mavromatos, "A Note on the Atiyah-Singer Index Theorem for Manifolds with Totally Antisymmetric H Torsion," J. Phys. A 21 (1988) 2279.

[37] C. M. Hull, "Compactifications of the Heterotic Superstring," Phys. Lett. B 178 (1986) 357.

[38] S. Ivanov and G. Papadopoulos, "A No-go Theorem for String Warped Compactifications," Phys. Lett. B 497 (2001) 309, hep-th/0008232.

[39] J. Gillard, G. Papadopoulos and D. Tsimpis, "Anomaly, Fluxes and $(2,0)$ Heterotic-string Compactifications," JHEP 0306 (2003) 035, hep-th/0304126.

[40] U. Gran, P. Lohrmann and G. Papadopoulos, "The Spinorial Geometry of Supersymmetric Heterotic String Backgrounds," JHEP 0602 (2006) 063, hep-th/0510176

[41] O. DeWolfe and S. B. Giddings, "Scales and Hierarchies in Warped Compactifications and Brane Worlds," Phys. Rev. D 67 (2003) 066008, hep-th/0208123.

[42] K. Becker and L. S. Tseng, "Heterotic Flux Compactifications and Their Moduli," Nucl. Phys. B 741 (2006) 162, hep-th/0509131.

[43] M. Nakahara, "Geometry, Topology and Physics," Institute of Physics Publishing (1990), Bristol. 
[44] S. Yajima, "Gravitational Anomalies with Curl Vanishing Torsion," Hiroshima University Preprint HUPD-8901 (1989).

[45] H. T. Nieh and M. L. Yan, "An Identity in Riemann-Cartan Geometry," J. Math. Phys. 23 (1982) 373.

[46] O. Chandia and J. Zanelli, "Topological Invariants, Instantons and Chiral Anomaly on Spaces with Torsion," Phys. Rev. D 55 (1997) 7580, hep-th/9702025.

[47] L. Alvarez-Gaumé, "Supersymmetry and the Atiyah-Singer Index Theorem," Commun. Math. Phys. 90 (1983) 161.

[48] L. Alvarez-Gaumé, "A Note on the Atiyah-Singer Index Theorem," J. Phys. A 16 (1983) 4177.

[49] K. Peeters and A. Waldron, "Spinors on Manifolds with Boundary: APS Index Theorems with Torsion," JHEP 9902 (1999) 024, hep-th/9901016.

[50] Y. N. Obukhov, "Spectral Geometry of the Riemann-Cartan Space-Time," Nucl. Phys. B 212 (1983) 237. 